EPJ Web of Conferences 41, 04020 (2013)

DOI: $10.1051 /$ epjconf/20134104020

C) Owned by the authors, published by EDP Sciences, 2013

\title{
Coherent phonon frequency comb generated by few-cycle femtosecond pulses in $\mathrm{Si}$
}

\author{
Muneaki Hase ${ }^{1,2}$, Masayuki Katsuragawa ${ }^{3}$, Anca Monia Constantinescu ${ }^{1}$, and Hrvoje Petek ${ }^{1}$ \\ ${ }^{1}$ Department of Physics and Astronomy, University of Pittsburgh, 3941 O’Hara Street, Pittsburgh, \\ PA 15260, USA \\ ${ }^{2}$ Institute of Applied Physics, University of Tsukuba, 1-1-1 Tennodai, Tsukuba 305-8573, Japan \\ ${ }^{3}$ Department of Engineering Science, University of Electro-Communications, 1-5-1 Chofugaoka, \\ Chofu, Tokyo 182-8585, Japan
}

\begin{abstract}
We explore the coherent phonon induced refractive index modulation of a $\mathrm{Si}(001)$ surface upon the excitation in near-resonance with the direct band gap of Si. Through the anisotropic $e-h$ pair generation and coherent Raman scattering, $\sim 10$-fs laser pulses exert a sudden electrostrictive force on Si lattice launching coherent LO phonon oscillations at $15.6 \mathrm{THz}$ frequency. The concomitant oscillatory change in the optical constants modulates the reflected probe light at the fundamental LO phonon frequency, generating a broad comb of frequencies at exact integer multiples of the fundamental frequency extending to beyond $100 \mathrm{THz}$. On the basis of an analytical model, we show that the simultaneous amplitude and phase modulation of the reflected light by the coherent lattice polarization at $15.6 \mathrm{THz}$ generates the frequency comb.
\end{abstract}

\section{Introduction}

The coherent modulation of Raman transition in gas phase atoms and molecules by intense few-cycle pulses has been used to generate Raman frequency combs that span multiple octaves $[1,2]$. Similar Raman frequency combs can be generated efficiently in solids on account of the high Raman polarizability densities. In the case of the Raman active phonon mode in solids, large amplitude coherent optical phonons could modulate light at multiple phonon frequencies in $\mathrm{THz}$ range.

In this paper, we demonstrate the phononic modulation of the optical index of Si for excitation and probing near its direct band gap $(\approx 3.4 \mathrm{eV})$ The large amplitude coherent longitudinal optical (LO) phonon of $\mathrm{Si}(001)$ excited by 10-fs laser pulses induces effective amplitude and phase modulation on the reflected probe light, generating a phonon frequency comb with more than 100 THz bandwidth.

\section{Experimental}

The anisotropic transient reflectivity of the $n$-doped $\left(1.0 \times 10^{15} \mathrm{~cm}^{-3}\right) \operatorname{Si}(001)$ sample was measured in air at $295 \mathrm{~K}$ by the electro-optic (EO) sampling technique [3]. Nearly collinear, pump and probe beams $\left[397 \mathrm{~nm}(3.12 \mathrm{eV})\right.$ center wavelength] were overlapped at a $7.2 \times 10^{-7} \mathrm{~cm}^{2}$ spot on the sample. The maximum average pump power from a $70 \mathrm{MHz}$ repetition rate, frequency doubled Ti:sapphire

This is an Open Access article distributed under the terms of the Creative Commons Attribution License 2.0, which permits unrestricted use, distribution, and reproduction in any medium, provided the original work is properly cited. 
laser oscillator of $60 \mathrm{~mW}$ generated $N \approx 1.0 \times 10^{20} \mathrm{~cm}^{-3}$ carriers. The electro-optic signal, $\Delta R_{\mathrm{EO}} / R_{0}$, versus the pump-probe delay was obtained by scanning a shaker at $20 \mathrm{~Hz}$ frequency and averaging the resultant traces over 20,000 times.

\section{Results and discussion}

Figure 1 shows the anisotropic transient reflectivity signal, $\Delta R_{\mathrm{EO}} / R_{0}$. Aperiodic electronic response near the zero time delay is observed, followed by a coherent oscillation with a time period of $\approx 64 \mathrm{fs}$ that persists for several picoseconds due to the zone-center coherent LO phonons $[4,5]$. As seen in Fig. 1, the $\Delta R_{\mathrm{EO}} / R_{0}$ signal can be fit approximately to a damped harmonic oscillation [5]. A finding uncovered here from the residual of the fit, shown in Fig. 1 inset, is that the 2-nd and higherharmonic oscillations (oscillation periods of 32, 21, and $16 \mathrm{fs}$, etc.) also contribute to the optical response of the Si sample.

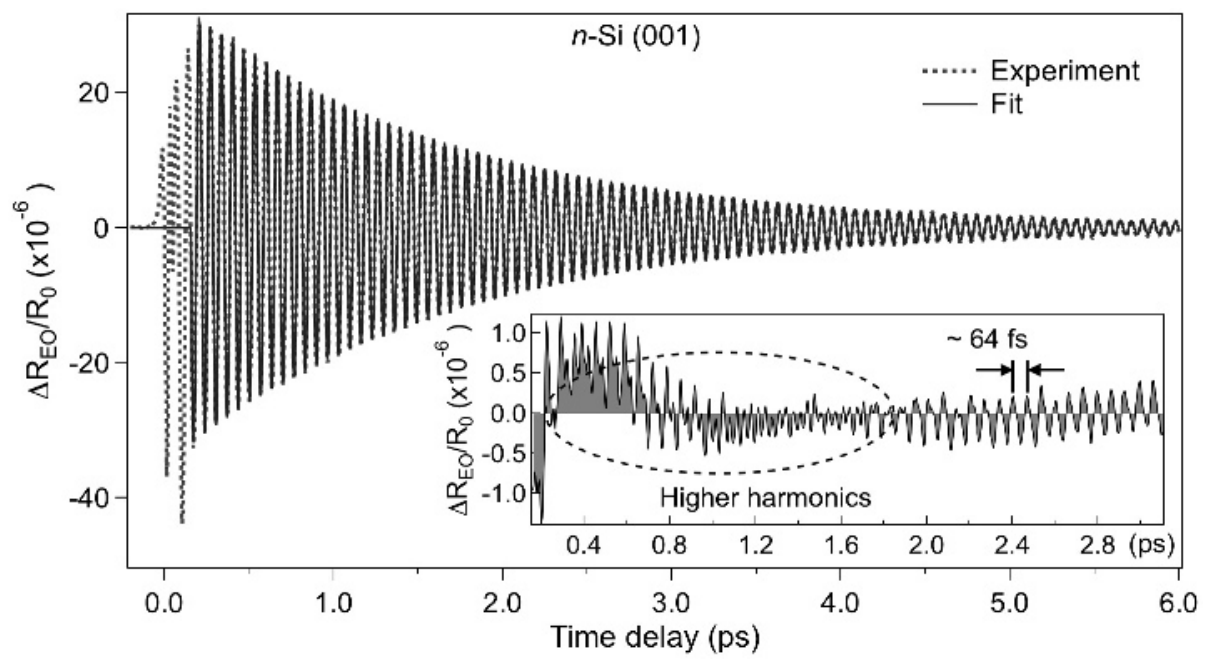

Fig. 1. Transient anisotropic reflectivity observed at the photoexcited carrier density of $N \approx 1.0 \times 10^{20}$ $\mathrm{cm}^{-3}$. The dashed line represents the experimental data and the solid line shows the fit with a damped harmonic oscillation. The inset is the residual of fit of the transient anisotropic reflectivity, revealing the higher harmonics of the coherent LO phonon oscillation.

To further investigate the higher-harmonics of the coherent LO phonon, we obtain the Fourier transformed (FT) spectrum, as shown in Fig. 2(a). It shows a comb of frequencies dominated by the fundamental LO phonon (15.6 THz) and its exact harmonics at 31.2, 46.8, 62.4, 78.0, 93.6, and 109.2 THz, up to the 7th-order.

The evenly spaced FT line spectrum cannot be attributed to the phonon ladder climbing [6] or the Raman overtone scattering [7], because these processes would produce anharmonic progressions. Our observation of the frequency comb is consistent with the excitation of $\mathrm{THz}$ Raman polarization, which modulates the optical constants of Si through the optical deformation potential, as can be explained by an analytical model below. The incident electric field of the probe is described by $E_{0} \cos \omega t$. In response to the $\mathrm{THz}$ Raman polarization, the longitudinal component of the reflected probe electric field $E_{\mathrm{Pr}}(t)$ experiences a phase and amplitude modulation [8],

$$
E_{\mathrm{Pr}}(t)=\left\{a(t) \cos \left(\omega_{\mathrm{LO}} t\right)+R_{0}\right\} E_{0} \cos \left[\omega t-\delta(t) \cos \left(\omega_{\mathrm{LO}} t+\varphi\right)\right],
$$


where $a(t) \cos \left(\omega_{\mathrm{LO}} t\right)$ describes the amplitude modulation (AM) and $\delta(t) \cos \left(\omega_{\mathrm{LO}} t+\varphi\right)$ describes the phase modulation (PM), with $a(t)=a_{0} e^{-t / \tau_{\mathrm{LO}}}$ and $\delta(t)=\delta_{0} e^{-t / \tau_{\mathrm{LO}}}$. Here, $\omega_{\mathrm{LO}}$ is the frequency and $\tau_{\mathrm{LO}}$ the relaxation time of the LO phonon. Other parameters are the static reflectivity, $R_{0}$, the optical carrier frequency, $\omega$, and the relative phase between the AM and PM, $\varphi$. The simulated FT spectrum based on Eq. (1) is displayed in Fig. 2(b). The evenly spaced frequency combs and asymmetric lineshapes could be reproduced well. Thus, the generation of the frequency comb can be attributed to the simultaneous actions of AM and PM on the probe electric field by the coherent LO polarization at $15.6 \mathrm{THz}[8]$.

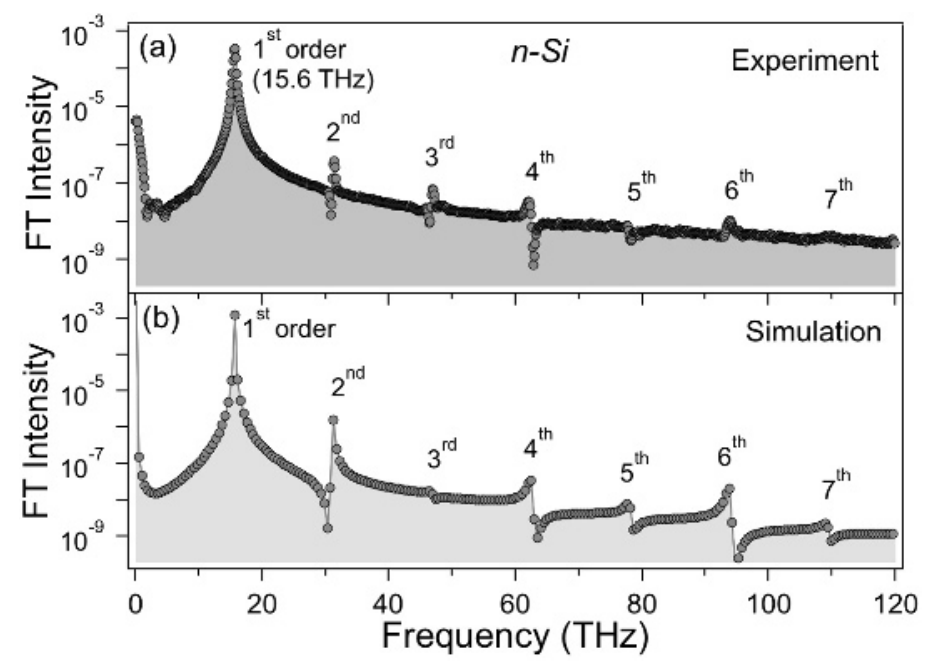

Fig. 2. (a) FT spectrum obtained from the anisotropic reflectivity in Fig. 1 at the photoexcited carrier density of $N \approx 1.0 \times 10^{20} \mathrm{~cm}^{-3}$. (b) Simulated spectrum based on the model, Eq. (1).

\section{Summary}

We have demonstrated ultrafast light modulation by excitation of large amplitude coherent LO phonons in Si using 10-fs ultrashort laser pulses. The $\mathrm{THz}$ polarization at the fundamental $\mathrm{LO}$ phonon frequency $(15.6 \mathrm{THz})$ modulates the amplitude and phase of the probe light through the optical deformation potential, thereby generating a frequency comb up to 7 th-order $(109.2 \mathrm{THz})$ of the driving frequency of the coherent LO phonon.

\section{References}

1. A. V. Sokolov, D. R. Walker, D. D. Yavuz, G. Y. Yin, and S. E. Harris, Phys. Rev. Lett. 85, 562-565 (2000).

2. T. Suzuki, M. Hirai, and M. Katsuragawa, Phys. Rev. Lett. 101, 243602 (2008).

3. G. C. Cho, W. Kütt, and H. Kurz, Phys. Rev. Lett. 65, 764-766 (1990).

4. A. J. Sabbah and D. M. Riffe, Phys. Rev. B 66, 165217 (2002).

5. M. Hase, M. Kitajima, A. M. Constantinescu, and H. Petek, Nature 426, 51-54 (2003).

6. C. J. Brennan and K. A. Nelson, J. Chem. Phys. 107, 9691-9694 (1997).

7. A. Bartels, T. Dekorsy, and H. Kurz, Phys. Rev. Lett. 84, 2981-2984 (2000).

8. M. Hase, M. Katsuragawa, A. M. Constantinescu, and H. Petek, Nature Photon. 6, 243-247 (2012). 